\title{
Development of an Emulator for the Plasma Process
}

\section{Control}

\author{
Marie-Pierre Planche, Taikai Liu, Sihao Deng and Ghislain Montavon \\ Research Institute on Transport, Energy and Society_Laboratory of Engineering Research on Materials, Processes and Surfaces, \\ University of Technology of Belfort-Montbeliard, Belfort 90010, France
}

Received: October 17, 2014 / Accepted: November 11, 2014 / Published: January 25, 2015.

\begin{abstract}
This work aims at developing an automatic system for the control of the APS (air plasma spraying) plasma process in which some instability phenomena are present. APS is a versatile technique to produce coatings of powder material at high deposition rates. Using this technique, powder particles are injected into a plasma jet, where they are melted and accelerated towards a substrate. The coating microstructures and properties depend strongly on the characteristics of the plasma jet, which can be controlled by the adjustment of the process parameters. However, the interactions among the spray variables, render optimization and control of this process are quite complex. Understanding relationships between coating properties and process parameters is mandatory to optimize the process technique and the product quality. We are interested in this work to build an on-line control model for the APS process based on the elements of artificial intelligence and to build an emulator that replicates the dynamic behavior of the process as closely as possible.
\end{abstract}

Key words: Atmospheric plasma spray, process parameters, artificial neural networks, fuzzy logic, emulator.

\section{Introduction}

Since its creation, the thermal spraying continuously expands its application scope because of its potential to project very different materials (metal, ceramic, plastic, etc.) as well as different forms (powder, wire, suspension, solution, etc.) [1-4]. Several types of methods have been developed to agree with industrial applications, such as the process HVOF (high velocity oxygen fuel) $[5,6]$, the process APS (atmospheric plasma spraying) [7-9] and the process VLPPS (very low pressure plasma spray) [10-12]. Among these methods, the APS process is now well established in the industry and laboratory for successfully developing coatings with good quality and relatively low cost [13-15]. However, this technology suffers from some instabilities leading to subjective product quality and endures a lack of

Corresponding author: Marie-Pierre Planche, associate professor, Ph.D., research fields: plasma process, artificial intelligence, diagnosis. E-mail: marie-pierre.planche@utbm.fr. understanding of the relationship between the operating parameters and the characteristics of in-flight particles. As a reminder, during the APS spraying, the arc root instability phenomena, the electrode erosion, the instability of the operating parameters cannot be completely eliminated $[16,17]$. Further, it is still difficult to correctly measure and control these parameters.

With the developing technology of diagnostic tools that can be used in a hostile environment (as in the case of APS process), an effective control of APS process in closed-loop can be considered and requires the development of an expert system consisting of ANNs (artificial neural networks) and FLC (fuzzy logic) controlling. The ANNs have been developed in several application fields and now also in plasma spraying area [18-20]. FLC controlling is an extension of boolean logic based on the mathematical theory of fuzzy sets [21-23].

In this work, the deal is first to build an on-line 
control model for the APS process based on the elements of artificial intelligence and second, to build an emulator that replicates the dynamic behavior of the process as closely as possible. Further, the ANNs will be combined with the emulator for constituting a big system which can monitor the process and also can automatically carry out modification action. The system then will be tested off-line. The paper is organized as follows: Section 2 discusses the process instabilities; Section 3 introduces the practical development of the automatic system; and Section 4 presents the emulator development; and Section 5 gives the conclusions.

\section{Process Instabilities and Their Consequences}

The principle of the plasma spraying is to create a high energy plasma capable to melt the injected powder and to spray it onto the surface before re-solidification. The hottest plasma regions near the anode output can reach $15,000{ }^{\circ} \mathrm{C}$ for a velocity of $2,000 \mathrm{~m} / \mathrm{s}$ [24]. In this type of d.c. (direct current) plasma torch, the arc strikes between the tip of the cathode and the concentric anode-nozzle. However, the superimposed flow influences the arc anode attachment column, thus, the arc exhibits a complex and unstable shape. As an immediate consequence, highly transient dynamic phenomena occur in arc voltage following by strong fluctuations in the plasma flow leading also to modifications in both the temperature and the velocity fields. In this context, the stability of the plasma is one of the most important factors for the quality of the sprayed coating since it leads to variations in particle heating, acceleration and trajectories.

Different instabilities in the d.c. plasma torch can be considered depending on their characteristic time as shown in Fig. 1:

- The first type concerns the electrode erosion with a characteristic time of $100 \mathrm{~s}$, and this progressive fluctuation induces a continuous decrease of the arc voltage;

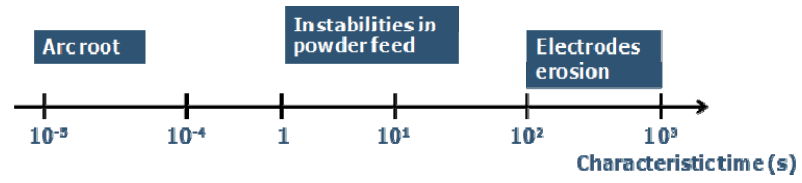

Fig. 1 Instabilities in d.c. plasma torch.

- The second type is related to the instabilities in the powder feed of about $1 \mathrm{~s}$ which leads to a homogeneous, discontinuous or pulsed distribution of the powder;

- The third type is around $10 \mathrm{~s}$ due to the arc root fluctuations which implies instantaneous variations of the electrical power and related plasma properties such as temperature and velocity.

The objective of this work is to develop an automatic system in order to at least stabilize the process and improve its reproducibility.

\section{Practical Development of the Automatic System}

Based on the on-line control via particle temperature and velocity, the schematic of the developed expert system is presented in Fig 2. Two aspects are combined in this approach mixing ANN for the data prediction and FLC for the process control.

In Fig. 2, gives the working of this automatic system step by step as follows:

- Considering a given coating property (porosity level for example) as input of the first ANN module (ANN 1), predicted particle temperature and velocity are then defined as output and finally used as input in the second ANN module (ANN 2) to predict operating parameters defined as output;

- The first FLC module (FLC 1) consists in getting back measured and predicted data in order to change operating parameters since the second FLC module (FLC 2) mixes ANN 2 output with FLC 1 output, comparing the respective values with the imposed ones in order to actually modify them.

The communication between data prediction and process control is transmitted via an appropriate file composed of different experimental results (Table 1). 


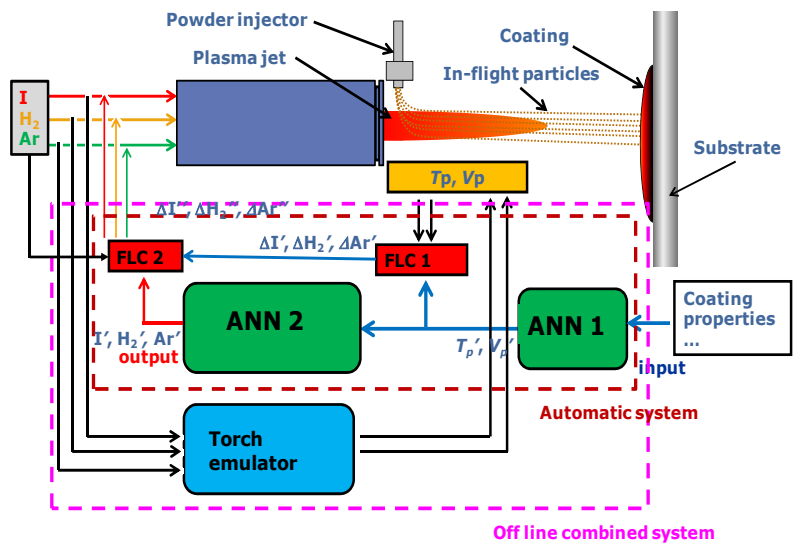

Fig. 2 Structure of the automatic system used for the process stabilization.

\subsection{ANN}

By definition, neural networks are networks of many simple processing units (called neurons) with dense parallel interconnections. Each neuron receives weighted inputs from other neurons and communicates its output to other neurons by an activation function. Neural network might be single or multilayer [25]. There is always an input layer with the number of neurons equal to the number of variables of the problem (in our case for operating parameters in Table 1) and an output layer with a number of neurons equal to desired number of quantities computed from the input (in our case for particle characteristics in Table 1). Layers (also called hidden layers) between input and output layers may contain a large number of hidden processing units. Most of the time, two hidden layers are required to be more efficient [26] and permit to justify our choice (Fig. 3).

In order to get the right number of neurons in the two hidden layers, a balance must be found between a great training efficiency and a great testing efficiency, meaning to balance between over-fit and under-fit training rules. Iterative process is performed to minimize the absolute percentage error values. Fig. 4 presents the best training and testing performances of ANN and the corresponding numbers of neurons determined for the two hidden layers of both ANN. Table 2 reports the found data.
Table 1 Variations of operating parameters and corresponding variations of particle temperature and velocity for $\mathrm{Al}_{2} \mathrm{O}_{3} / 13 \% \mathrm{TiO}_{2}$ powder.

\begin{tabular}{|c|c|}
\hline \multicolumn{2}{|c|}{ Operating parameters } \\
\hline Current intensity (A) & $350-750$ \\
\hline Argon flow rate (L/min) & $34-52$ \\
\hline Hydrogen flow rate $(\mathrm{L} / \mathrm{min})$ & $0-15$ \\
\hline \multicolumn{2}{|c|}{ Particle characteristics at $100 \mathrm{~mm}$ from the nozzle exit } \\
\hline In-flight velocity $(\mathrm{m} / \mathrm{s})$ & $150-400$ \\
\hline In-flight temperature $\left({ }^{\circ} \mathrm{C}\right)$ & $2,000-3,000$ \\
\hline
\end{tabular}

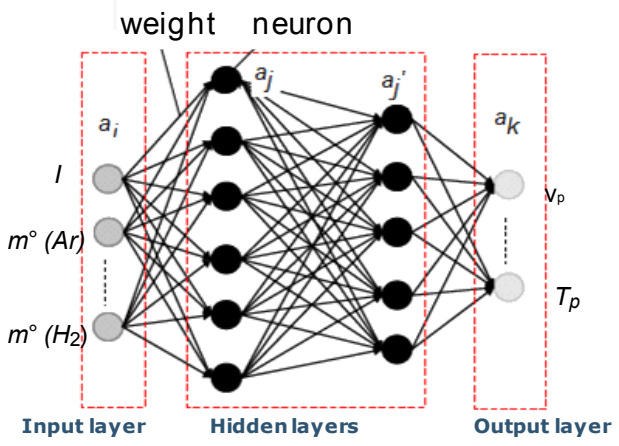

Fig. 3 Architecture of ANN.
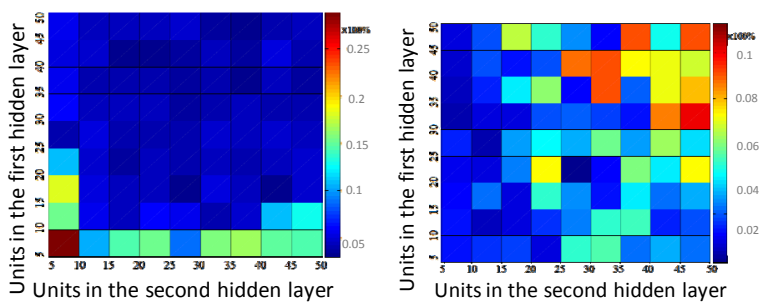

Fig. 4 Performances of ANN 1 and ANN 2: (a) training performance attesting by the blue color, and (b) testing performance attesting by the blue color.

Table 2 Numbers of neurons in the first and second hidden layers for ANN 1 and ANN 2.

\begin{tabular}{lll}
\hline & First hidden layer & Second hidden layer \\
\hline ANN 1 & 15 & 5 \\
ANN 2 & 15 & 10 \\
\hline
\end{tabular}

The trained model was then tested with the input values and the results were close to experiment values corresponding to particle temperature and velocity measured by the DPV-2000 (Tecnar, Canada). The training and the testing sets seem to be acceptable for ANN 1 focused on the particle characteristics prediction since the average error is less than 5\% (Fig. 5a). Concerning the ANN 2 dedicated to the operating parameters prediction, the average error is under $1 \%$ for the current intensity, $6 \%$ for the $\mathrm{H}_{2}$ flow rate and 
$4 \%$ for the Ar flow rate which concludes the acceptance of this model again (Fig. 5b).

\subsection{FLC}

The FLC system is a powerful system for the simulation of nonlinear behaviors with the help of FLC and linguistic rules [27]. This system is based on an expertise expressed from fuzzy rules. That means to determine selecting relevant input variables, to determine the number of fuzzy rules, their antecedents and consequents, to determine also the type of inference functions. These membership functions can be viewed as graphical representation of the magnitude of the contribution of each input on the considered result. Then, FLC can be decomposed into five successive steps (Fig. 6):

(1) Uniformization of the input variables;

(2) Reduction of input variables into linguistic descriptions (fuzzification step);

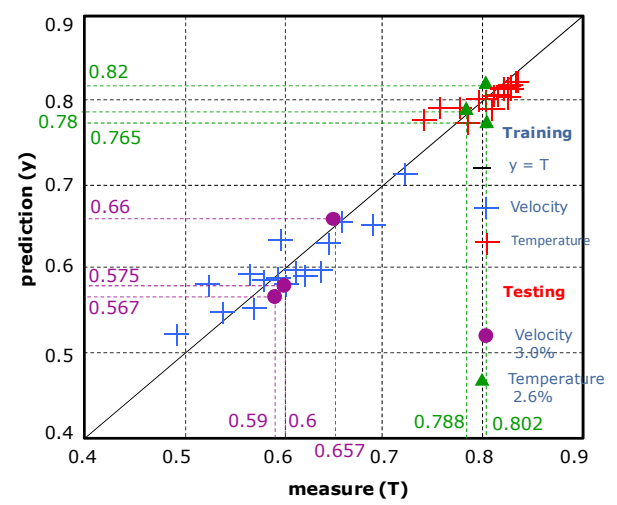

(a)

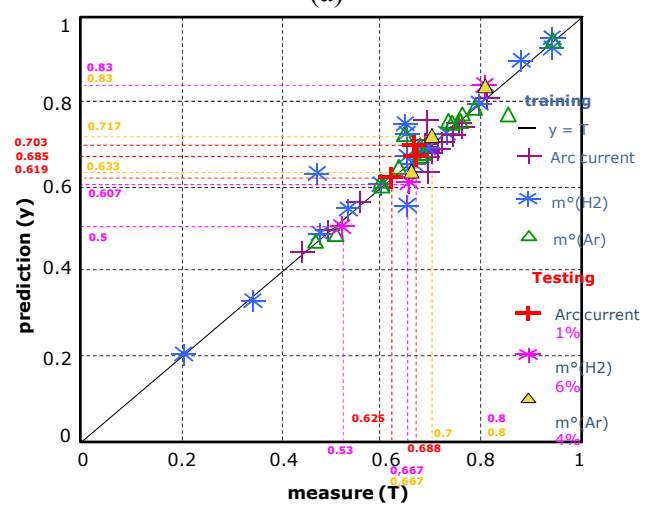

(b)

Fig. 5 (a) Validation of particle characteristics prediction with ANN 1 and (b) validation of operating parameters prediction with ANN 2.

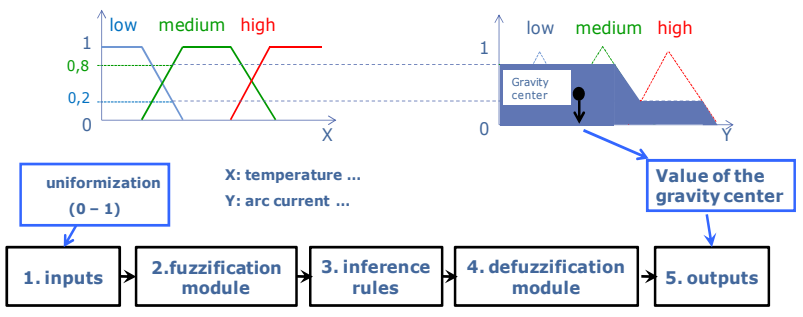

Fig. 6 FLC methodology.

(3) Application of the fuzzy rules via the inference module; fuzzy rules were defined from experimental data and employ fuzzy "if...then" rules;

(4) Translation of linguistic data into mathematical data (defuzzication step);

(5) Conversion of the mathematical data into output variables taking into account the value of gravity center.

In this model, the number of degrees was fixed at 5 because it gives the best deal between the number of fuzzy rules, the model accuracy and its response. Concerning the type of level, the output variables were determined by a triangular-shaped function because of its sensibility whereas the trapezoid-shaped was used for input variables because of its flexibility (Fig. 7).

Based on these choices, the number of fuzzy laws is then imposed by both numbers of input variables and levels being affected by each variable. Two input variables (in flight particle temperature and velocity) compose the first module FLC 1 which gives rise to $5^{2}$ fuzzy laws whereas the three independent input variables (current intensity, $\mathrm{Ar}$ and $\mathrm{H}_{2}$ flow rates) in the second module (FLC 2) permit to reduce the number of fuzzy laws from $5^{3}$ to $5^{1}$. In relation with these definitions, the consequences of imposed fuzzy laws can be verified in plotting the graphs representing the corresponding areas (Fig. 8 for FLC 1 and Fig. 9 for FLC 2, respectively).

As it can be observed, the area of FLC 1 corresponds to the difference between measured and predicted particle characteristics: a positive difference indicates that the measured value is higher than the predicted one. This way, the FLC1 module can rapidly get an inference. 


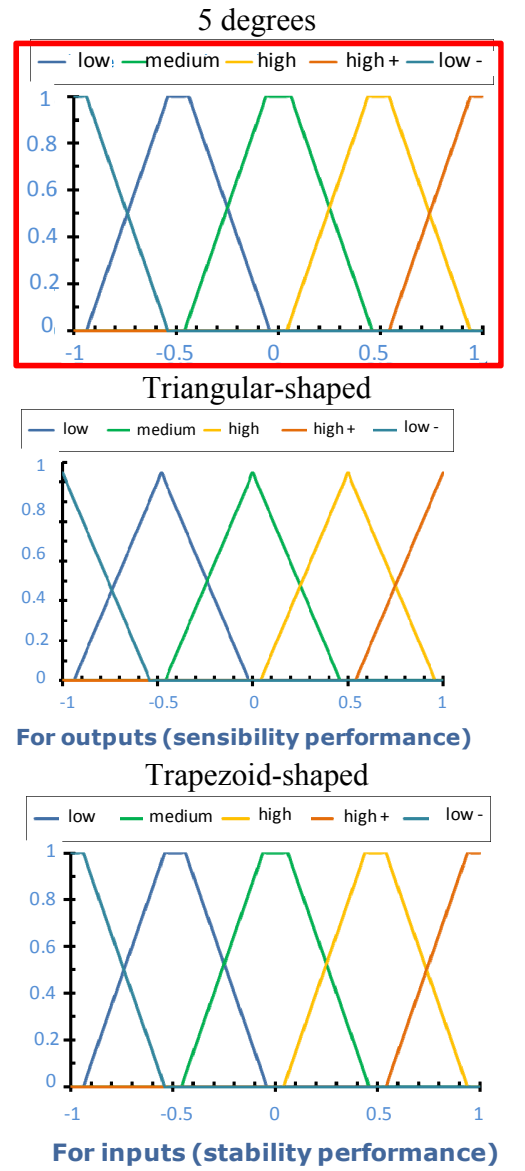

Fig. 7 Illustration of the number of degrees and type of functions.

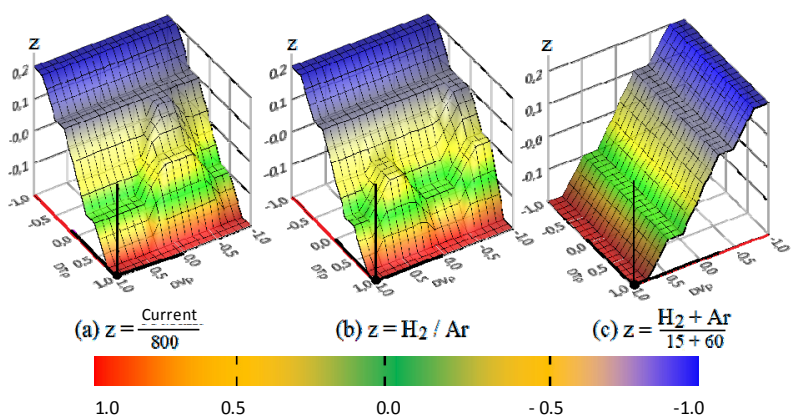

Fig. 8 Areas of fuzzy laws used in FLC 1 module giving velocity and temperature vs. operating parameters.

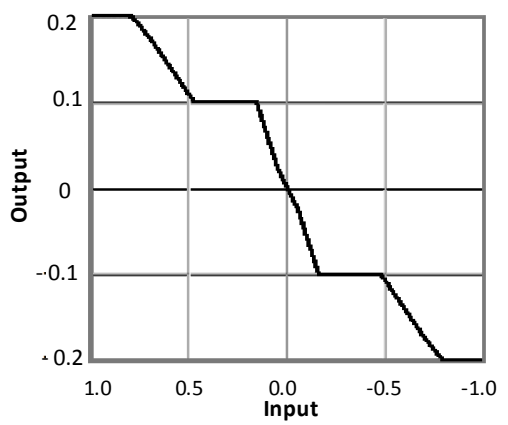

Fig. 9 Profile of fuzzy laws used in FLC 2 module.
Concerning the FLC 2 module, each operating parameter is considered independent and corresponds to the difference between the measured value and the reference value provided by the ANN 2 module and the operating parameters used in FLC 1 module. This way, a positive value implies to increase the operating parameters.

\subsection{Expert System}

The two ANN modules are then combined with the two FLC modules in order to get the expert system. The interface of this expert system is developed using Labview.

The structure of the expert system is depicted in Fig. 10.

Its training has been performed from experimental data (those measured on line by DPV-2000 and those found by coating analyses). This part was already reported in Ref. [28]. According to the obtained results previously detailed in Sections 1 and 2, it has been proved that this expert system succeeds in the data prediction and the off-line process control.

\section{Emulator Development}

\subsection{Emulator Parameters}

The emulator is based on the identification system which can be described as a measure of both the stimuli and the responses of the process. In the context of this study, the stimuli correspond to the operating parameters on-line measured and the responses are the in-flight particle characteristics also on-line measured by the DPV-2000 (Fig. 11).

Concerning the identification system, it can be used

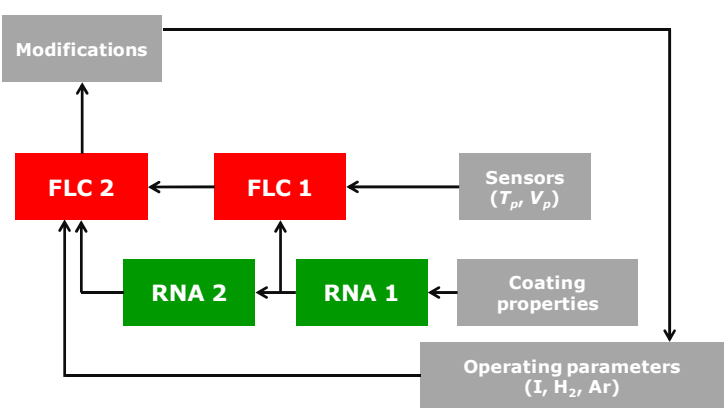

Fig. 10 Structure of the expert system. 


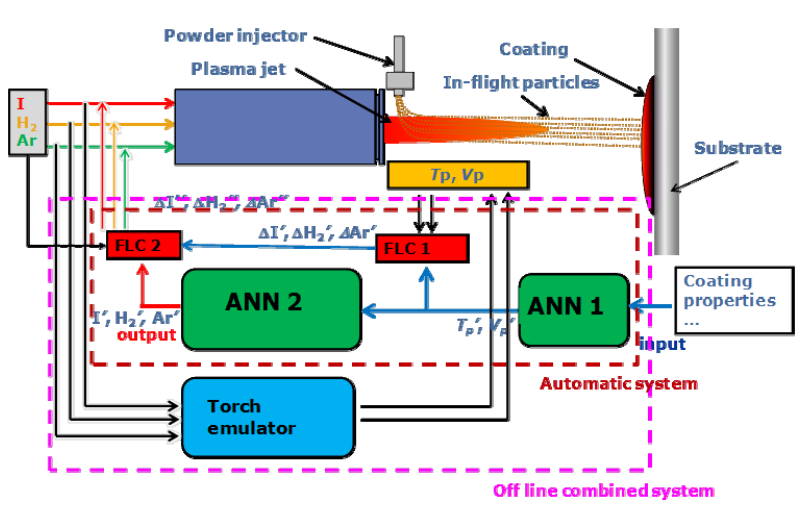

Fig. 11 Working of the emulator.

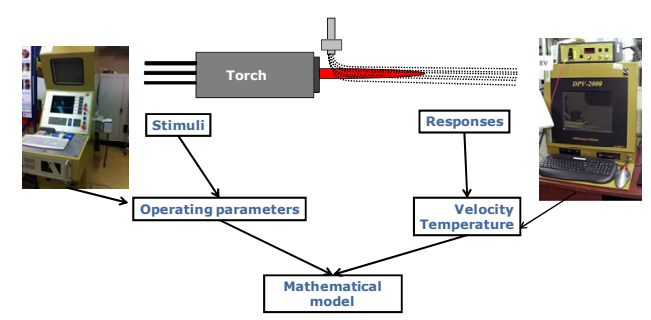

Fig. 12 Development of the identification system.

for building the mathematical model of the dynamic system, as presented in Fig. 12. This system precisely described in Ref. [29] uses the NLARX (nonlinear auto-regressive external) approach for properly simulating the d.c. plasma torch. The emulator was firstly trained with experimental data imported in Matlab and secondly implanted under the Labview platform. The experimental data are divided into two groups: one for the operating parameters and the other for in-flight characteristics.

In this mathematical model based on NLARX principle, it is important to define the following parameters:

- The first one is called "delay" which represents the time interval between the input and the output;

- The second one is called "number of terms" which is directly related to the number of inputs.

Different calculations were performed to find the best compromise between these two parameters. As proved in Fig. 13, the best one is obtained for a delay of 3 and for a number of terms of 5. Indeed, the prediction accuracy of temperature and velocity increase to reach a maximum of $71 \%$ and $80 \%$, respectively.
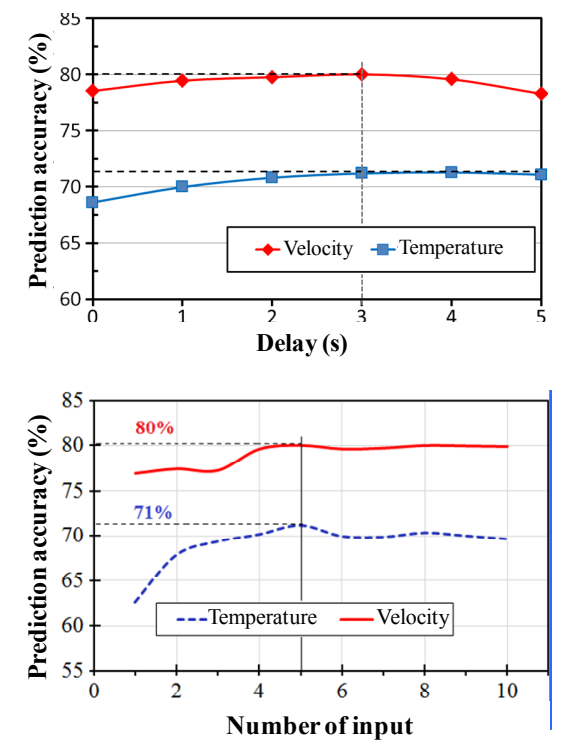

Fig. 13 Choice of the emulator parameters.

\subsection{Performances of the Emulator}

Thanks to this coefficients optimization, the prediction error of temperature and velocity can be estimated (Fig. 14).

It can be noticed that an average prediction error is around $2.5 \%$ and $5 \%$ for the temperature and the velocity respectively when taking into the complete duration account. That confirms the efficiency of NLARX model in correctly modeling the plasma torch process.

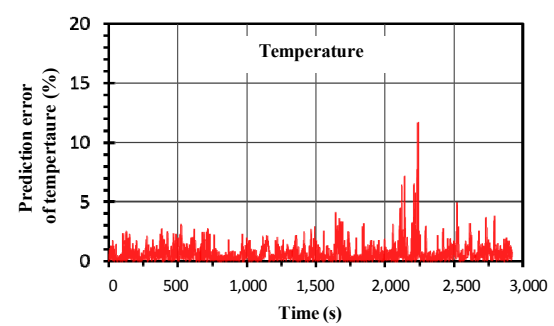

(a)

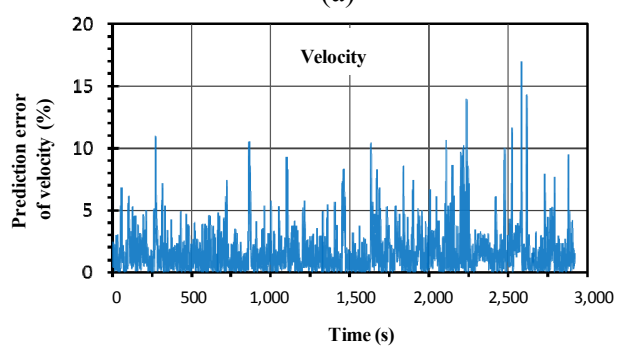

(b)

Fig. 14 Prediction of error (\%) of (a) temperature and (b) velocity. 


\subsection{Emulator Interface}

The NLARX model was trained using Matlab and then transferred into Labview in order to elaborate the graphical programming. The main advantage of this programming comes from its facilities to be connected to the AI (artificial intelligence) system. Indeed, its outputs can be used by the AI system whereas its inputs can be replaced by the AI system outputs. This way, the off line system can be built by combining the emulator with the AI system. Fig. 15 depicts the interface of this programming. Considering given inputs, both screens show the emulator response for temperature and velocity, respectively.

Finally, the association of both expert system and emulator workings give rise to a so called "combined system" using graphical interface and Matlab code. This system allows monitoring the plasma process and then automatically modifies the operating parameters on the basis of those predicted otherwise. This complete development is presented in Fig. 16.

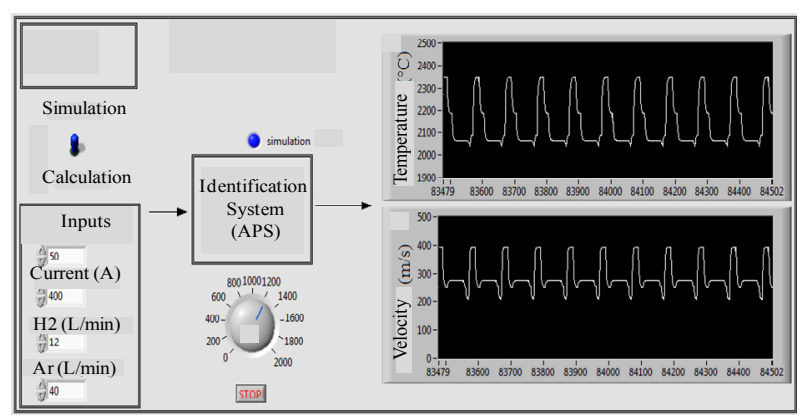

Fig. 15 Interface of the emulator.

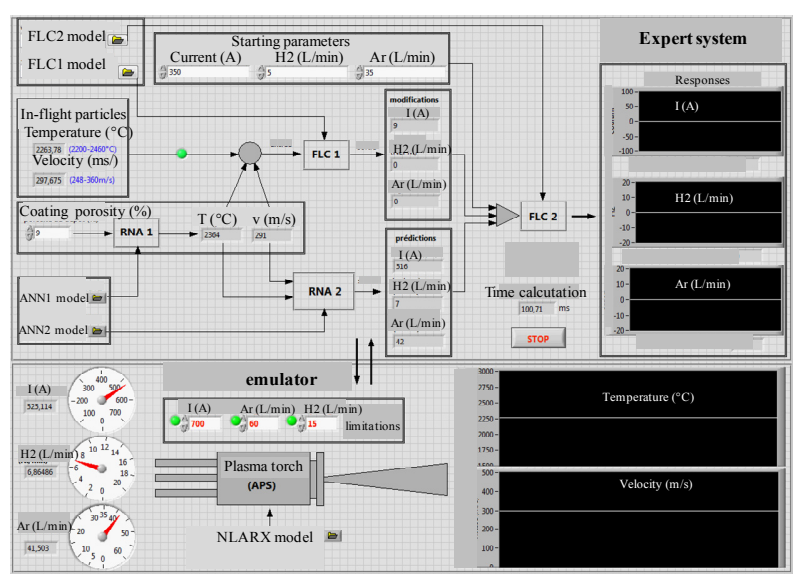

Fig. 16 Interface of the combined system.
Fig. 16 depicts the inputs of both inputs for expert system and emulator. The successive stages of this combined system operation can be followed as:

- Porosity level imposed;

- ANN and FLC modules worked;

- Modifications notified into outputs and used as emulator inputs;

- Temperature and velocity values changed in emulator outputs;

- Emulators outputs used as FLC 1 inputs.

\section{Conclusions}

A control system of the plasma process was developed using Labview software by combining ANNs and FLC. ANNs can predict speed and temperature of the particles in flight with a pretty low average error $(<3 \%)$, it also correctly predicts the operating parameters with an error of $1.2 \%$ for the intensity current, $3.9 \%$ for the flow rate of argon and $6.4 \%$ for the hydrogen flow.

The FLC modules are designed to monitor the process and indicate the changes to be performed on the operating parameters to match the predictions given by the ANNs. After training this system developed from experimental data (those measured), the expert system is able to predict the fair values and realize automatic control of plasma process (currently offline).

Then, an identification system established on the basis of a NLARX model was designed to simulate the operation of the plasma torch. A Matlab tool was chosen to drive the emulator (process simulator) with experimental data previously interpolated. The emulator simulates the temperature and velocity of the particles in flight with a percentage of acceptance of $72 \%$ and $83 \%$ respectively and an average difference simulation of $1 \%$ and $2 \%$, accordingly. After this training phase, the emulator is implemented in Labview to perform system monitoring and process control.

By combining the emulator with the expert system 
developed in Labview, a combined system (graphical, Matlab code) is obtained which allows to monitor the plasma process and automatically change settings based on those from the prediction.

\section{References}

[1] Cinca, N., Lima, C., and Guilemany, J. 2013. "An Overview of Intermetallics Research and Application: Status of Thermal Spray Coatings." Journal of Materials Research and Technology 2 (1): 75-86.

[2] Dorfman, M. R. 2012. Thermal Spray Coatings. 2nd edition. New York: Handbook of Environmental Degradation of Materials.

[3] Dosta, S., Torrell, M., Cano, I. G., and Guilemany, J. M. 2012. "Functional Colored Ceramic Coatings Obtained by Thermal Spray for Decorative Applications.” Journal of the European Ceramic Society 32 (14): 3685-92.

[4] Siegmann, S., and Abert, C. 2013. "100 Years of Thermal Spray: About the Inventor Max Ulrich Schoop." Surface and Coatings Technology 220: 3-13.

[5] Hong, S., Wu, Y., Zheng, Y., Wang, B., Gao, W., and Lin, J. 2013. "Microstructure and Electrochemical Properties of Nanostructured WC-10Co-4Cr Coating Prepared by HVOF spraying." Surface and Coatings Technology 235 (25): 582-8.

[6] Ni, L., Wu, Z., and Zhou, C. 2011. "Effects of Surface Modification on Isothermal Oxidation Behavior of HVOF-Sprayed NiCrAlY Coatings." Progress in Natural Science: Materials International 21 (2): 173-9.

[7] Richer, P., Yandouzi, M., Beauvais, L., and Jodoin, B. 2010. "Oxidation Behaviour of CoNiCrAlY Bond Coats Produced by Plasma, HVOF and Cold Gas Dynamic Spraying." Surface and Coatings Technology 204 (24): 3962-74.

[8] Syed, A., Denoirjean, A., Hannoyer, B., Fauchais, P., Denoirjean, P., Khan, A., and Labbe, J. C. 2005. "Influence of Substrate Surface Conditions on the Plasma Sprayed Ceramic and Metallic Particles Flattening." Surface and Coatings Technology 200 (7): 2317-31.

[9] Bobzin, K., Bagcivan, N., and Petkovic, I. 2011. "Numerical and Experimental Determination of Plasma Temperature during Air Plasma Spraying with a Multiple Cathodes Torch." Journal of Materials Processing Technology 211 (10): 1620-8.

[10] Yang, D., Tian, B., and Gao, Y. 2013. "The Lamellar-Equiaxed Microstructural Transition of $316 \mathrm{~L}$ Coatings by Low Pressure Plasma Spraying." Surface and Coatings Technology 228: S72-6.

[11] Zhu, L., Zhang, N., Zhang, B., Sun, F., Bolot, R., Planche, M. P., Liao, H., and Coddet, C. 2011. "Very Low Pressure Plasma Sprayed Alumina and Yttria-Stabilized
Zirconia Thin Dense Coatings Using a Modified Transferred Arc Plasma Torch.” Applied Surface Science 258 (4): 1422-8.

[12] Vautherin, B., Planche, M. P., Bolot, R., Quet, A., Bianchi, L., and Montavon, G. 2014. "Vapor and Droplets Mixture Deposition of Metallic Coatings by Very Low Pressure Plasma Spraying." Journal of Thermal Spray Technology 23 (4): 596-608.

[13] Gadow, R., Killinger, A., and Stiegler, N. 2010. "Hydroxyapatite Coatings for Biomedical Applications Deposited by Different Thermal Spray Techniques." Surface and Coatings Technology 205 (4): 1157-64.

[14] Fauchais, P., Montavon, G., Vardelle, M., and Cedelle, J. 2006. "Developments in Direct Current Plasma Spraying." Surface and Coatings Technology 201 (5): 1908-21.

[15] Zhang, T., Gawne, D., and Liu, B. 2010. "Computer Modeling of the Influence of Process Parameters on the Heating and Acceleration of Particles during Plasma Spraying." Surface and Coatings Technology 132 (4): 949-53.

[16] Coudert, J. F., and Rat, V. 2000. "The Role of Torch Instabilities in the Suspension Plasma Spraying Process." Surface and Coatings Technology 205 (2-3): 233-43.

[17] Coudert, J. F., Planche, M. P., and Fauchais, P. 1994. "Anode Arc Attachment in a Spray Plasma Torch." High Temperature Chemical Processes 3: 639-51.

[18] Guessasma, S., Montavon, G., and Coddet, C. 2003. "Neural Network, Design of Experiments and Other Optimization Methodologies to Quantify Parameter Dependence of Atmospheric Plasma Spraying." In Proceedings of the International Thermal Spray Conference (Thermal Spray 2003), 939-48.

[19] Kanta, A. F., Montavon, G., Berndt, C., Planche, M. P., and Coddet, C. 2011. "Intelligent System for Prediction and Control: Application in Plasma Spray Process." Expert Systems with Applications 38 (1): 260-71.

[20] Kanta, A. F., Montavon, G., Vardelle, M., Planche, M. P., Berndt, C. C., and Coddet, C. 2008. "Artificial Neural Networks vs. Fuzzy Logic: Simple Tools to Predict and Control Complex Processes-Application to Plasma Spray Processes." Journal of Thermal Spray Technology 17: $365-76$

[21] Ogaji, S., Marinai, L., Sampth, S., Singh, R., and Prober, S. 2005. "Gas Turbine Fault, Diagnostics: A Fuzzy-Logic Approach.” Applied Energy 32 (1): 81-9.

[22] Lee, C. 1990. "Fuzzy Logic in Control Systems: Fuzzy Logic Controller." IEEE Transactions on Systems, Man and Cybernetics 20 (2): 404-35.

[23] Hajek, P. 2006. "What Is Mathematical Fuzzy Logic?" Fuzzy Sets and Systems 157 (5): 597-603.

[24] Moreau, C., Gougeon, P., Lamontagne, M., Lacase, V., 
Vaudreuil, G., and Cielo, P. 2006. "On-line Control of Plasma Spraying Process by Monitoring the Temperature, Velocity and Trajectory of the In-flight Particles." In Proceeding of 7th National Thermal Spray Conference, 431-7.

[25] Mohamed, M. 2011. "Performance of Fuzzy Logic and Artificial Neural Network in Prediction of Ground and Air Vibrations." International Journal of Rock Mechanics and Mining Sciences 48: 845-51.

[26] Kuo, R. 2000. "Multi-sensor Integration for On-line Tool Wear Estimation through Artificial Neural Networks and Fuzzy Neural Network." Engineering Applications of Artificial Intelligence 13: 249-61.
[27] Mamdani, E. 1976. "Advances in the Linguistic Synthesis of Fuzzy Controllers." International Journal of Man-Machine Studies 8 (6): 669-78.

[28] Kanta, A. F. 2007. "Development of an Expert System Based on Artificial Intelligence to Predict and Control Plasma Spray Processes." M.Sc. thesis, University of Franche-Comte.

[29] Liu, T., Planche, M. P., Deng, S., and Montavon, G. 2014. "Optimization of an Emulator Based on Nonlinear Auto Regressive Model with Exogenous Inputs for an Atmospheric Plasma Spray Torch." Presented at the IEEE Conference on Intelligent Transportation System, Barcelona, Spain. 